\title{
Minimizing Disagreements in the United Nations
}

\author{
Alexander Soifer
}

\begin{abstract}
I would like to present here an example of a bridge between problems of mathematics and problems of mathematical Olympiads. Graph Theory has been a fertile ground for extracting beautiful ideas that would work very well in the Olympiad-type competitions. The problem presented here served as problem 4 in the $27^{\text {th }}$ Colorado Mathematical Olympiad that took place on April 23, 2010.
\end{abstract}

Key words and phrases: Graph theory, mathematical olympiads, problems, disagreements.

\section{Minimizando Desacuerdos en las Naciones Unidas \\ Resumen}

Me gustaría presentar aquí un ejemplo de un puente entre problemas de matemática y problemas de olimpiadas matemáticas. La Teoría de Grafos ha sido campo fértil para extraer hermosas ideas que pueden trabajarse muy bien en competencias de tipo olimpiadas. El problema aquí presentado se usó como problema 4 en la 27a Olimpiada Matemática de Colorado, que tuvo lugar el 23 de abril del año 2010.

Palabras y frases clave: Teoría de Grafos, olimpiadas matemáticas, problemas, desacuerdos.

\section{Introduction}

In order to create this problem, I needed to know how many member countries were in the United Nations. I guessed 200 - and was quite close!

Wikipedia informs:

“TheUnited Nations Organization (UNO) or simply United Nations (UN) is an international organization whose stated aims are facilitating cooperation in in- 
ternational law, international security, economic development, social progress, human rights, and the achieving of world peace... There are currently $192 \mathrm{mem}$ ber states, including nearly every sovereign state in the world."

And now the problem:

"In Order to Form a More Perfect Union..."(A. Soifer, 2010). The United Nations Organization includes 192 Member States, every pair of which has a disagreement. In order to form a more perfect Union, a negotiation is introduced: ifrepresentatives of four Member States are seated at a round table so that each pair of neighbors has a disagreement, the negotiation resolves one of these four disagreements. A series of consecutive negotiations reduces the total number of disagreements to $n$. What is the minimum of $n$ ?

\section{Solution.}

1) Let each Member Country be represented by a vertex of a graph, in which we connect two vertices by an edge if and only if the corresponding countries have a disagreement. Then the Initial Disagreements Graph is the complete graph $K_{192}$ on 192 vertices (a set of 192 vertices, every two of which are connected by an edge). A negotiation selects a 4-cycle $C_{4}$ of a graph (remember: "representatives of four countries are seated at a round table so that each pair of neighbors has a disagreement") and removes one edge from it. The problem, translated into this language, asks to find the minimum number of edges in a Disagreements Graph obtained from the initial $K_{192}$ by a series of consecutive removals of an edge from a 4-cycle.

2) Observe that the removal of an edge in a $C_{4}$ subgraph preserves connectivity of a graph (i.e., ability to travel between any pair of points through a series of edges).

If the series of consecutive negotiations were to eliminate all cycles, we would get a connected cycle-free graph, called a tree, on 192 vertices. Such a tree has exactly 191 edges (proof by an easy induction is left for the pleasure of the readers).

Observe that for any two points of a tree we have a unique path connecting them through a series of edges (for otherwise we would have created a cycle contained in the union of two distinct paths). This observation allows us to show that any tree is 2colorable (so that vertices of the same color are not adjacent to each other). Indeed, color a point $\mathrm{A}$ in color $\mathrm{0}$, and any other point $\mathrm{B}$ in color 0 or 1 depending upon the parity of the edge distance from A to B.

Observe now that the property of 2-colorability is preserved under the operation converse to negotiation, i.e., the operation of completing a 4-path to a 4-cycle. Therefore, if we assume that the graph with 191 edges is achieved, the Initial Disagreements Graph is also 2-colorable. However, the Initial Disagreements Graph $K_{192}$ is not 2-colorable (in fact, it requires 192 colors!), therefore we will never get a tree as a result of a series of negotiations! We proved that 191 edges (i.e., disagreements) is unreachable.

3) On the other hand, we can fly a kite and in the process get a Disagreements Graph with 192 edges. 


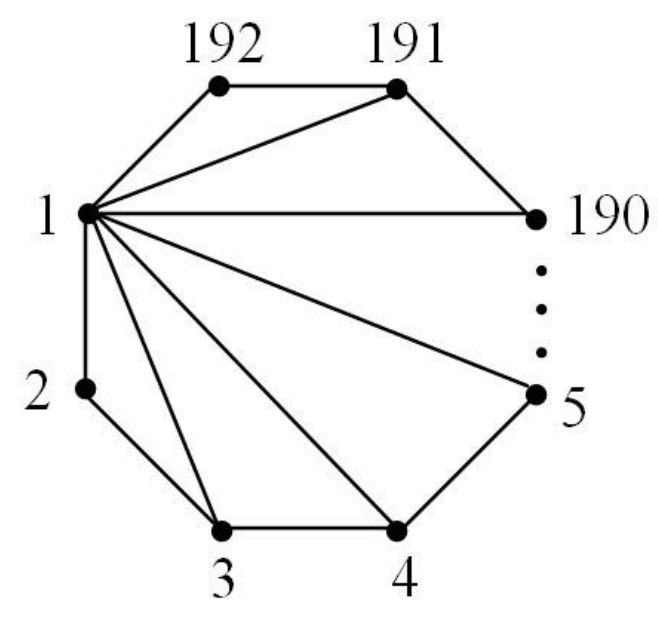

Figure 1: Kite-0, which is $K_{192}$ where only edges emanating from vertex 1 are shown

Through the series of negotiations we can get from the Kite- 0 graph, which is $K_{192}$, to the Kite-1 graph, which consists of $K_{191}$ with an attached 1-edge "tail." Indeed (see Figure 1$)$, from the 4 -cycle $\{1,3,4,5\}$ we remove the edge $\{1,3\}$; from $\{1,4,5,6\}$ remove $\{1,4\} ; \ldots$, from $\{1,190,191,192$ remove $\{1,190\}$; from $\{1,191,192,2\}$ remove $\{1,191\}$. Finally, from the 4-cycle $\{1,2,3,192\}$, we remove $\{1,192\}$, getting the desired graph Kite-1 (Figure 2).

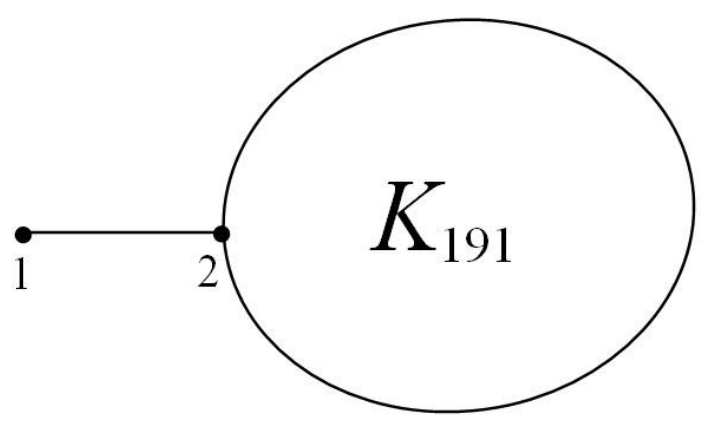

Figure 2: Kite-1, which is $K_{191}$ with a 'tale' $1-2$.

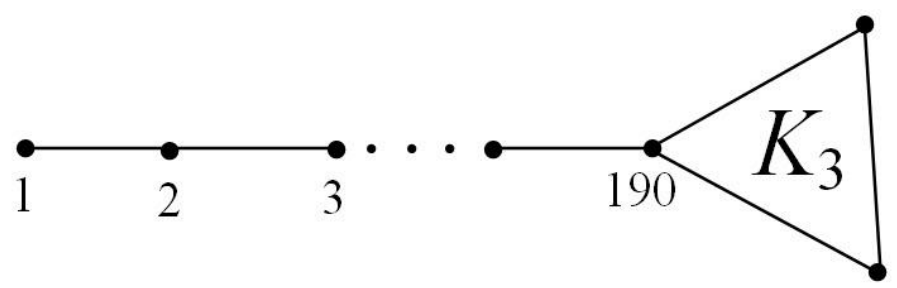

Figure 3: Kite-189, which is $K_{3}$ with a tale 189 -edges long 
Continuing this process (you can formalize it by a simple induction), we will get to Kite-189graph, which consists of $K_{3}$ with a tail of length 189 (figure 3), which has exactly 192 edges as desired.

\section{Homework for Children \& Consenting to Homework Adults.}

Determine which of the graphs in Figures 4 and 5 can be obtained from the Initial Disagreements Graph $K_{192}$ through a series of negotiations.

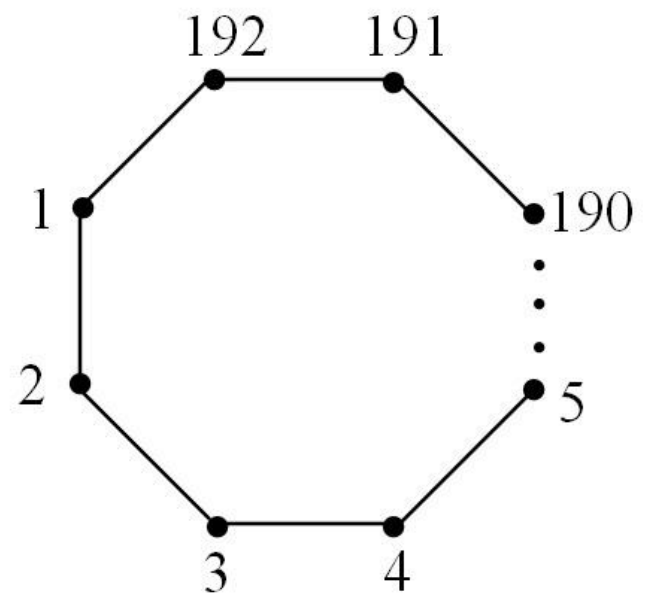

Figure 4: Cycle $C_{192}$ on 192 vertices

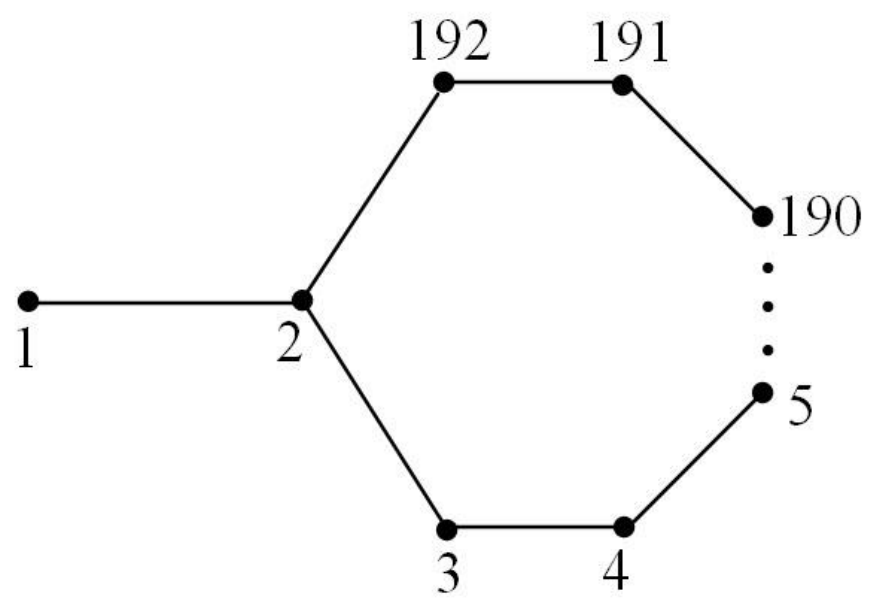

Figure 5: Cycle $C_{192}$ on 191 vertices with a 'tale' 1-2

\section{Alexander Soifer (asoifer@uccs.edu) \\ University of Colorado, Colorado Springs}

